\title{
A comparison of the sensitivity and specificity of sialodacryoadenitis virus, Parker's rat coronavirus, and mouse hepatitis virus-infected cells as a source of antigen for the detection of antibody to rat coronaviruses
}

\author{
D. H. Percy ${ }^{1}$, K. L. Williams ${ }^{1}$, and F. X. Paturzo ${ }^{2}$ \\ ${ }^{1}$ Department of Pathology, Ontario Veterinary College, University of Guelph, Guelph, \\ Ontario, Canada \\ ${ }^{2}$ Section of Comparative Medicine, Yale School of Medicine, Yale University, New Haven, \\ Connecticut, U.S.A.
}

Accepted January 7, 1991

Summary. Sialodacryoadenitis virus (SDAV) and Parker's rat coronavirus (PRC) are two recognized viral strains which cause spontaneous disease in the laboratory rat. Currently there is no recognized practical procedure which will accurately differentiate infections with these strains. Using SDAV- and PRCinfected L-2 cells as the source of antigen, and sera from rats collected post inoculation with either of these viral strains, the indirect fluorescent antibody (IFA) procedure was used to determine whether antibody titers could be used to differentiate infections from the homologous and heterologous virus. There was no detectable difference in the sensitivity or specificity of these systems in detecting antibody to the homologous or heterologous virus. Thus there was no evidence that SDAV- and PRC-infected cells would serve to differentiate antibody to the homologous virus using the IFA technique. In addition, antibody titers were similar when mouse hepatitis virus ( $\mathrm{MHV}$ )-infected cells were used as the source of antigen for the IFA technique. However, using MHV or SDAV-infected cells as the source of antigen, there was a significant difference in antibody titers to the homologous virus detected using the immunoenzyme technique.

\section{Introduction}

The indirect fluorescence antibody (IFA) test is a recognized, sensitive method to detect antibody to specific rodent viruses. One method used for the detection of coronaviral antibodies in rodent sera consists of equal proportions of cells infected with the JHM and S strains of mouse hepatitis virus (MHV) as a source of antigen, together with uninfected cells. Using this as a source of antigen, the indirect fluorescent antibody procedure has proven to be a test system with 
good sensitivity for the detection of animals seropositive for murine coronaviruses [6]. Similarly, enzyme immunoassay (EIA) procedures have been developed for antibody detection and titration. Using the horseradish peroxidase or the urease assay, antibody titers may be substantially higher than those detected with the IFA technique [7].

Using the L-2 subline of L-929 cells, we have been able to replicate relatively high titers of sialodacryoadenitis virus (SDAV) [4], and Parker's rat coronavirus (PRC) [5] in vitro. The purpose of this study was to compare the sensitivity of SDAV or PRC-infected L-2 cells with MHV-infected cells for the detection and titration of antibody to SDAV or PRC. The techniques used were IFA and an immunoenzyme procedure.

\section{Materials and methods}

\section{Cells}

The L-2 subline of L-929 cells for the replication of SDAV or PRC was obtained from Dr. V. L. Morris, University of Western Ontario, London, Ontario. MHV-infected NCTC 1469 cells, prepared as previously described for L-929 cells [6] were acquired from Dr. A. L. Smith, Section of Comparative Medicine, Yale School of Medicine.

\section{Virus}

The \#681 strain of SDAV (family Coronaviridae) was obtained from Dr. P. N. Bhatt, Yale School of Medicine. The isolate of PRC (family Coronaviridae) was obtained from the American Type Culture Collection, Rockville, MD. SDAV and PRC were replicated in L2 cells as previously described $[4,5]$. For the preparation of viral antigen from rat coronaviruses, L-2 cells were propagated in $100 \times 15 \mathrm{~mm}$ Nunc polystyrene tissue culture dishes (Gibco/BRL Inc, Burlington, Ont.) in Eagle's minimal essential medium (Gibco/BRL Inc). Cells were grown in a humidified atmosphere containing $5 \% \mathrm{CO}_{2}$. When approximately $80 \%$ confluent, monolayers were inoculated with stock virus suspension, and incubated for $48 \mathrm{~h}$ at $37^{\circ} \mathrm{C}$ in $5 \% \mathrm{CO}_{2}$. Infected and control cells were then removed using a rubber policeman, placed in two separate centrifuge tubes, and centrifuged at $1600 \mathrm{rpm}$, then resuspended in PBS. Cells were then mixed at one part of infected to 1.5 parts of noninfected cells. $20 \mu \mathrm{l}$ of the mixture were then dropped on each well in multiwell depression glass slides (Gibco/BRL Inc). Slides were air dried for $1 \mathrm{~h}$, fixed in acetone, and stored at $-70^{\circ} \mathrm{C}$ until tested.

\section{Animal inoculation and sera collections}

SPF Wistar rats were obtained at approximately 8 weeks of age from a commercial supplier (Charles River Laboratories, St. Constant, Quebec). Animals were housed in an isolation facility throughout the inoculation period. In separate studies and at different time periods, rats were inoculated intranasally with approximately $10^{3} \mathrm{TCID}_{50}$ of either the ATCC strain of PRC or strain \#681 of SDAV (second passage in L-2 cells). Animals were killed with an overdose of pentobarbitone sodium (MTC Pharmaceuticals, Mississauga, Ont.) at 6, 8, 14 , and 21 days post-inoculation (pi), sera were collected by cardiac puncture, and tissues were collected from control and inoculated animals for histopathology.

\section{Indirect fluorescence antibody procedure}

Sera were tested for antibody titers using the following virus-infected cells as antigens: SDAV, PRC, and MHV. Serum samples were diluted in PBS, then individual wells were 
flooded with the appropriate dilution and processed as previously described [6]. Fluorescein-labelled anti-rat globulin (Antibodies Inc., Davis, CA) was used to identify positive samples. Evans blue at $0.005 \%$ was used as a counterstain. Specimens were examined using a halogen-illuminated epifluorescence microscope at $\times 250$ and $\times 400$ magnifications. All samples were read as unknowns to avoid bias. Titers were recorded as the highest dilution of the serum sample under test showing fluorescence. The differences between the mean values for the antibody titers to the homologous and heterologous viruses in each study were tested for significance using the general linear model of SAS (GLM).

\section{Enzyme immunoassay technique}

For the immunoenzyme technique, sera collected from spontaneous cases of SDA were used to evaluate the sensitivity of MHV and SDAV-infected cells for antibody titration. The procedure, using horse radish peroxidase-conjugate anti-rat IgG, has been previously described [7].

\section{Results}

Typical changes $[2,8]$ observed in the PRC- and SDAV-inoculated rats at necropsy. In the SDA virus-infected L-2 cells prepared for the IFA technique, viral antigen was frequently demonstrated in the cytoplasm of syncytial giant

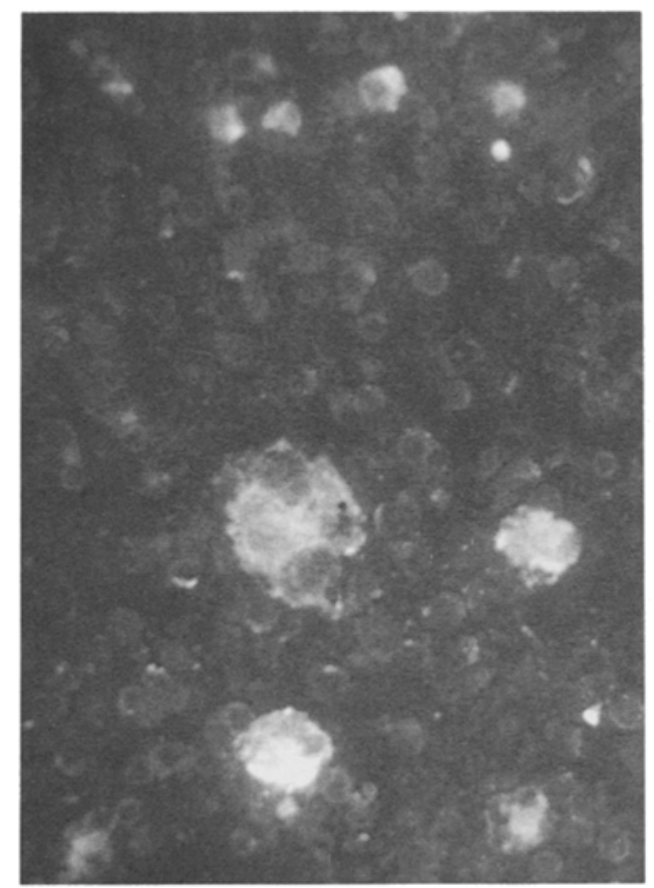

a

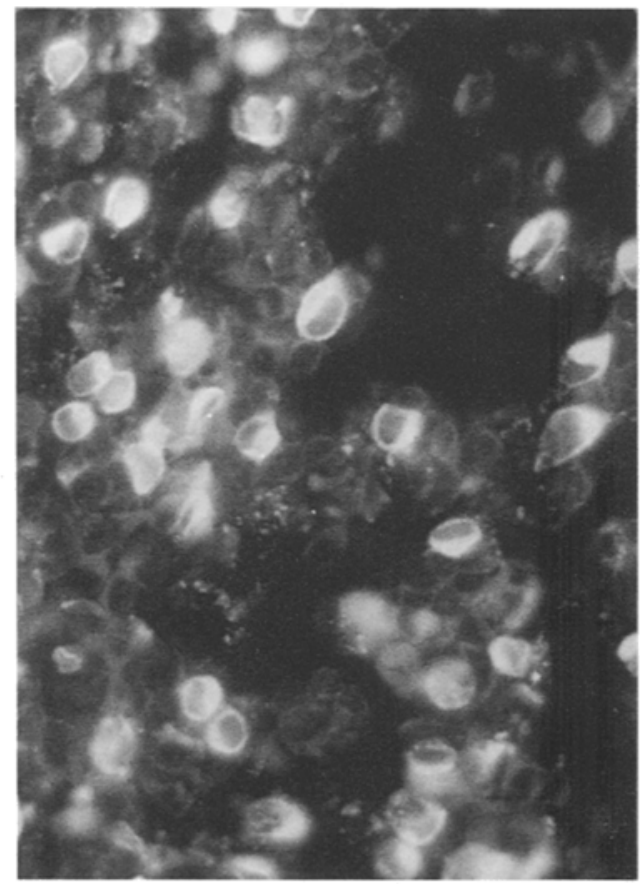

b

Fig. 1. a SDAV-infected L-2 cells stained by the indirect immunofluorescence microscopy (IFA) technique, using convalescent serum from a rat post exposure to SDAV. Intracytoplasmic fluorescence is particularly marked in syncytial cells. $\times 250$. b PRC-infected L-2 cells stained using the IFA technique and convalescent serum collected from a rat post exposure to SDAV. Viral antigen is evident primarily in the cytoplasm of individual cells. 
cells (Fig. 1 a). With L-2 cells inoculated with PRC and prepared for the IFA techniqe, cytoplasmic fluorescence was present most frequently in single or binucleated cells (Fig. 1 b).

\section{Indirect fluorescence antibody procedure}

Using the IFA procedure and MHV-, SDAV-, or PRC-infected cells as a source of antigen and sera from rats exposed to SDA virus, antibody titers to all three antigens were similar (Table 1). There was no statistically-significant difference in antibody titers to the three antigens $(\mathrm{p}<0.0001)$. Similarly, in a comparison of antibody titers in rats inoculated with PRC, antibody titers were similar (Table 2). There was no significant difference in the mean antibody titers to the homologous virus compared to the heterologous viral antigens $(p<0.0001)$.

\section{Enzyme immunoassay procedure}

Using MHV-infected NCTC cells and SDAV-infected L-2 cells as the source of antigen, serum antibody titers of SDAV-infected rats were usually substantially higher with homologous antigen than with the MHV-infected cells (Table 3). In a comparison of the mean antibody titers, the antibody titers to

Table 1. Comparison of antibody titers in rats exposed to sialodacryoadenitis virus using SDAV, PRC, and MHV-infected cells (indirect immunofluorescence microscopy)

\begin{tabular}{lrrr}
\hline & \multicolumn{3}{c}{ Antigen (virus) } \\
\cline { 2 - 4 } Sample no. & SDAV $^{\mathrm{a}}$ & PRC $^{\mathrm{b}}$ & MHV $^{\mathrm{c}}$ \\
\hline 1 & & & \\
2 & $640^{*}$ & 480 & 320 \\
3 & 2560 & 2560 & 640 \\
4 & 640 & 640 & 1280 \\
5 & 80 & 40 & 40 \\
6 & 40 & 40 & 40 \\
7 & 80 & 40 & 80 \\
8 & 80 & 80 & 80 \\
9 & 160 & 160 & 160 \\
10 & 80 & 80 & 80 \\
11 & 160 & 160 & 160 \\
\hline
\end{tabular}

${ }^{\text {a }}$ Sialodacryoadenitis virus

${ }^{\text {b }}$ Parker's rat coronavirus

${ }^{\mathrm{c}}$ Mouse hepatitis virus

* No significant difference in the means of the antibody titers to the three viral antigens $(p<0.0001)$
Table 2. Comparison of antibody titers in rats exposed to Parker's rat coronavirus using SDAV, PRC, and MHV-infected cells (indirect immunofluorescence microscopy)

\begin{tabular}{lrrr}
\hline & \multicolumn{3}{c}{ Antigen (virus) } \\
\cline { 2 - 4 } Sample no. & SDAV & PRC & MHV \\
\hline 6 & & & \\
7 & $0^{*}$ & 0 & 0 \\
8 & 640 & 320 & 640 \\
13 & 640 & 320 & 320 \\
15 & 40 & 40 & 40 \\
16 & 320 & 320 & 320 \\
20 & 320 & 320 & 640 \\
9 & 640 & 640 & 320 \\
10 & 80 & 80 & 40 \\
11 & 80 & 80 & 80 \\
12 & 80 & 80 & 80 \\
\hline
\end{tabular}

* No significant difference in the means of the antibody titers to the three viral antigens $(\mathrm{p}<0.0001)$ 
Table 3. Comparison of antibody titers in rats exposed to SDAV using SDAV and MHV-infected cells (enzyme immunoassay method)

\begin{tabular}{llr}
\hline & \multicolumn{2}{l}{ Antigen (virus) } \\
\cline { 2 - 2 } Sample no. & SDAV & MHV \\
\hline 5 & & \\
6 & $2560^{*}$ & 1280 \\
7 & 2560 & 640 \\
8 & 2560 & 640 \\
9 & 2560 & 1280 \\
10 & 5120 & 640 \\
11 & 1280 & 2560 \\
12 & 2560 & 1280 \\
13 & 2560 & 640 \\
14 & 5120 & 640 \\
\hline
\end{tabular}

* The mean of the antibody titers to the homologous virus (SDAV) is significantly higher than to MHV $(\mathrm{p}<0.0046)$

the homologous viral antigen were significantly higher than those detected using the heterologous virus $(\mathrm{p}<0.0046)$.

\section{Discussion}

The ability to replicate relatively high titers of rat coronaviruses in a continuous cell line has provided the opportunity to evaluate the sensitivity and specificity of rat coronaviral-infected cells compared to MHV-infected cells as a source of antigen for the detection and titration of antibody to SDAV and PRC. The antigenic similarities among the rodent coronaviruses have been emphasized in previous studies [1,3], thus allowing the use of MHV-infected cells as a source of antigen for the detection of antibodies to either mouse or rat coronaviruses [6]. Based on our studies, the use of rat coronavirus-infected cells as a source of antigen offers no significant advantage over MHV-infected cells. Furthermore, we were unable to demonstrate any appreciable advantage in using either SDAV or PRC as a source of viral antigen to detect antibody to the homologous virus, either in sensitivity or specificity. Based on these data, there is no evidence that IFA staining of L-2 cells infected with either SDAV or PRC will serve to differentiate antibody to the homologous or heterologous virus. However, using MHV or SDAV-infected cells as a source of antigen for the enzyme immunoassay procedure, there was a significant difference in the anti-SDAV antibody titers using the homologous virus $(\mathrm{p}<0.0046)$. SDA antibody titers were usually considerably higher using the enzyme immunoassay procedure with homologous antigen than with $\mathrm{MHV}$-infected cells. 


\section{Acknowledgements}

This work was supported by Natural Sciences Research Council grant \#A0071, the Ontario Ministry of Agriculture and Food, and USPHS grant RR00393. The technical assistance of Ted Eaton and Jean Bagg are gratefully acknowledged. Special thanks to Dr. Jeff Wilson, Population Medicine, University of Guelph, for the statistical analyses.

\section{References}

1. Bhatt PN, Percy DH, Jonas AM (1972) Characterization of the virus of sialodacryoadenitis of rats: a member of the coronavirus group. J Infect Dis 126: 123-130

2. Jacoby RO, Bhatt PN, Jonas AM (1979) Coronaviruses. In: Baker HJ, et al (eds) The laboratory rat. Biology and diseases. Academic Press, New York, pp 284-294

3. Parker JC, Cross SS, Rowe WP (1970) Rat coronavirus (RCV): a prevalent, naturallyoccurring pneumotropic virus of rats. Arch Ges Virusforsch 31: 293-302

4. Percy DH, Bond SJ, MacInnes JI (1989) Replication of sialodacryoadenitis virus in mouse L-2 cells. Arch Virol 104: 323-333

5. Percy DH, Williams KL, Bond SJ, MacInnes JI (1990) Characteristics of Parker's rat coronavirus (PRC) replicated in L-2 cells. Arch Virol 112: 195-202

6. Smith AL (1983) An immunofluorescence test for detection of serum antibody to rodent coronaviruses. Lab Anim Sci 33: 157-160

7. Smith AL, Winogard DF (1986) Two enzyme immunoassays for detection of antibody to rodent coronaviruses. J Virol Methods 14: 335-343

8. Wojcinski ZW, Percy DH (1986) Sialodacryoadenitis virus-associated lesions in the lower respiratory tract of rats. Vet Pahtol 23: 278-286

Authors' address: D. H. Percy, Department of Pathology, Ontario Veterinary College, University of Guelph, Guelph, Ont. N1G 2W1, Canada.

Received November 4, 1990 